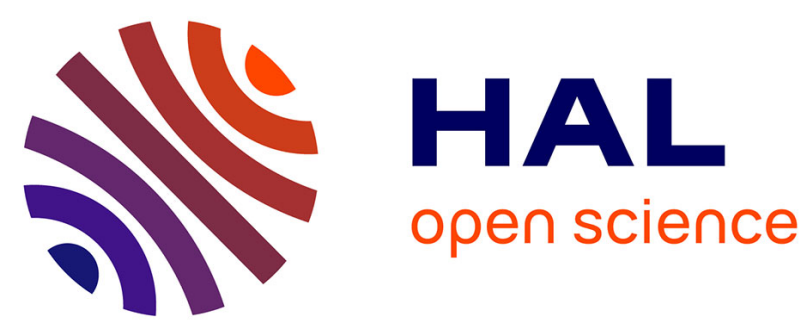

\title{
Application-aware cost function and its performance evaluation over scalable video conferencing services on heterogeneous networks
}

Tien A. Le, Hang Nguyen

\section{- To cite this version:}

Tien A. Le, Hang Nguyen. Application-aware cost function and its performance evaluation over scalable video conferencing services on heterogeneous networks. 2012 IEEE Wireless Communications and Networking Conference: Mobile and Wireless Networks (IEEE WCNC 2012 Track 3 Mobile

Wireless), citeulike-article-id = 10124426, Apr 2012, Paris, France, France. pp.1-6. hal-00703013

\section{HAL Id: hal-00703013 \\ https://hal.science/hal-00703013}

Submitted on 31 May 2012

HAL is a multi-disciplinary open access archive for the deposit and dissemination of scientific research documents, whether they are published or not. The documents may come from teaching and research institutions in France or abroad, or from public or private research centers.
L'archive ouverte pluridisciplinaire HAL, est destinée au dépôt et à la diffusion de documents scientifiques de niveau recherche, publiés ou non, émanant des établissements d'enseignement et de recherche français ou étrangers, des laboratoires publics ou privés. 


\title{
Application-aware cost function and its performance evaluation over scalable video conferencing services on heterogeneous networks
}

\author{
Tien Anh Le, Hang Nguyen
}

\begin{abstract}
Video conferencing service requires a multicast tree to distribute its multimedia contents to all participants. Link cost is very important in building such media distribution trees. In this research work, a multi-variable cost function is proposed. This cost function can calculate links' costs based on both network resources and application's requirements. Since participants may join the conference using either a high speed wireless network such as WiMAX or fixed (heterogeneous) networks, we construct a scalable video conferencing service on an overlay network of a simulated Internet topology and a real WiMAX network and apply the newly proposed cost function for building the multimedia distribution tree for the service. Some participants join the conference from the WiMAX network and others from the Internet. An intensive evaluation platform has been built to evaluate the performance of the newly proposed cost function. The collected real measurement data have validated the advanced performance of the new cost function in the rapidly changing heterogeneous network environment.
\end{abstract}

Index Terms - video conference service; application layer multicast routing; cost function; 4G; WiMAX; heterogeneous network; field tests and measurements

\section{INTRODUCTION}

Multi-party video conferencing service is the most complicated type of communication because of its many-tomany nature. In order to build a video conference service on the Internet, a multicast mechanism is required. The Internet was originally built for unicast or one-to-one applications. Nowadays, it has to serve a large number of multimedia services such as video streaming, multimedia conference. These types of multicast services put a big load on the unicast infrastructure of the Internet. Therefore, a multicast mechanism is required in order to serve the modern many-to-many multimedia services on the Internet. IP-Multicast[1] is the first attempt to solve this problem. However, many deploying problems are still preventing IP-Multicast from being supported worldwide[2]. An alternative solution is Application Level Multicast(ALM). The key concept of ALM is the implementation of multicasting functionality as an application service instead of a network service. It has excellent advantages over IPMulticast: easier and possibly immediate deployment over the Internet without any modification of the current infrastructure and adaptable to a specific application. In a tree-push ALM, a data distribution tree is built first, then

Authors are with the Department of Wireless Networks and Multimedia Services, Telecom Sud Paris, 91011, France. Phone: +33(0)160 $76 \quad 66$ 63, Fax: +33(0)160 $76 \quad 45$ 78, E-mail: \{Tien_Anh.Le, Hang.Nguyen\}@it-sudparis.eu. This work was supported in part by POSEIDON, a French national project on multimedia services over $4 \mathrm{G}$ networks. the data is actively distributed from the source node to intermediate peers until reaching all peers in the multicast tree[3]. In order to build an ALM distribution tree, we must have costs of all available end-to-end links. Those costs can only be calculated by using a cost function.

The contributions of this research are:

- Design a new multi-variable cost function of the endto-end delay and bandwidth taking into account advantages of application layer links,

- Propose an evaluation platform for the scalable video conference service built from the new cost function,

- Collect real measurement data for validating the advanced performance of the new cost function in the rapidly changing heterogeneous network environment.

The research work is an extension to the original work proposed in[4][5]. After having proposed the new multivariable cost function, it is necessary to evaluate its performance. Since it has been proved that SVC-content can resist better in the heterogeneous environment of the overlay network[6][7], therefore an evaluation platform of the newly proposed multi-variable cost function with SVC content is highly required. The general architecture of the evaluation platform is demonstrated in Fig.1.

Video conferencing service requires a multicast tree to distribute its multimedia contents to all participants. Link cost is very important in building such media distribution trees. Since participants may join the conference using either a high speed wireless network such as WiMAX or fixed (heterogeneous) networks, we construct a scalable video conferencing service on an overlay network of a simulated Internet topology and a real WiMAX network and apply the newly proposed cost function for building the multimedia distribution tree for the service. Some participants join the conference from the WiMAX network and others from the Internet. An intensive evaluation platform has been built to evaluate the performance of the newly proposed cost function. The collected real measurement data have validated the advanced performance of the new cost function in the rapidly changing heterogeneous network environment.

We have built an extended evaluation platform from our original evaluation platform for scalable video transmission (EvalSVC[8]). This platform provides measurement data of a scalable video conference service on heterogeneous environment of real WiMAX network with real WiMAX BSS, real WiMAX core network, and two participants joining the conference using two real WiMAX terminals (more details in subsection III.A) and a simulated Internet topol- 


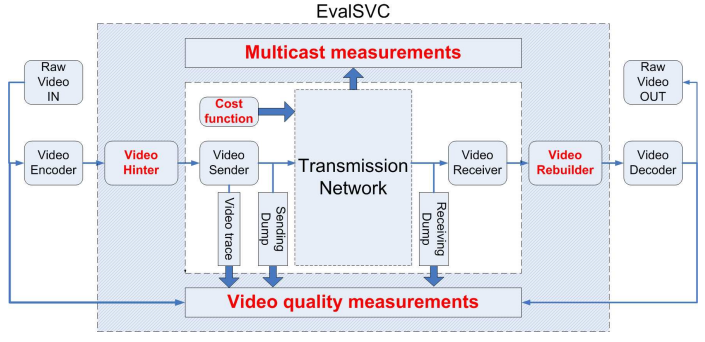

Fig. 1. EvalSVC and the evaluation of SVC-based services on overlay network constructed by the newly proposed multi-variable cost function.

ogy with up to one thousand participants. The collected measurements have shown the adaptability of the new cost function in such a fast changing conditions as of heterogeneous networks.

The rest of the paper is organized as follows. The proposed multi-variable cost function will be described and derived in section II. In subsection III.A, the settings of the evaluation platform are shown. The measurement results are then given and analyzed in subsection III.B. Conclusion and future work are in section V.

\section{Multi-VARiable COST function}

Conventional cost functions are either empirical or heuristic. Among all available cost functions for ALM routing that we have found, neither of them has a mathematical derivation nor a clear citation. In most of the ALM routing algorithms, the state of the network, on which the routing algorithm is presented, readily associates some costs with each link. Thus they do not address how the link cost function should be defined so as to efficiently distribute allocated resources over the network[9][10][11][12]. This again raises needs for a new multi-variable cost function. Assuming that we have an overlay with application peers and end-to-end-links, in order to form a tree for data delivery, we need costs of all those end-to-end links. These costs must be calculated by a cost function. To take into account several QoS parameters simultaneously, the cost function must be a multi-variable function. QoS parameters can be a bandwidth-type (meaning that the requested bandwidth is always smaller than or equal to the maximum available bandwidth) or delay-type (meaning that the requested delay is always greater than or equal to the minimum available delay). On each end-to-end link, we have to consider variable requirements from applications running on the P2P-based overlay. For example, an application can be a scalable video service with different video coding layers or it can be a multimedia flux comprising of video, audio, text, data sub-streams, each has different bandwidth and delay requirements. Those requirements are changed frequently by the application. We have to also consider the maximum available resources of the underlay. For example, if an end-to-end link is built upon 3 physical links, each has its own available bandwidth and delay. Then the maximum available bandwidth of the end-to-end link equals to the minimum available bandwidth (bottle- neck) of all 3 physical links, the minimum guaranteed delay of the end-to-end link equals to the sum of all delays on the 3 physical links.

\section{A. Problem formation}

Problem: Find a multi-variable cost function which can simultaneously consider varied bandwidth and delay requests from the application and maximum guaranteed resources from the underlay network. The cost function must be able to assign increasingly higher costs for nearlysaturated end-to-end links to prevent congestion.

\section{B. Single variable cost function}

Assume we have on the end-to-end link $i$ : A total available bandwidth of $\kappa_{w}$, and a requested bandwidth of $x_{w}$, we must find the bandwidth-type cost function: $f\left(x_{w}\right)$. Since $\kappa_{w}$ is the maximum available bandwidth when using all available resources on link $i$, so $0 \leq x_{w} \leq \kappa_{w}$. With time, according to the application's requirements, $x_{w}$ may be varied by an amount of $\Delta x_{w}$ causing the cost to have the current value of $f\left(x_{w}+\Delta x_{w}\right)$, so this current value of the cost function depends on:

- The previous cost: $f\left(x_{w}\right)$,

- The increment of cost which is proportional to:

- The previous cost: $f\left(x_{w}\right)$,

- The ratio between the increment of requested bandwidth and the total requested bandwidth: $\frac{\Delta x_{w}}{x_{w}+\Delta x_{w}}$,

- The decrement of cost which is proportional to:

- The ratio between the decrement of the remaining available bandwidth and the maximum available bandwidth $\frac{\left(\kappa_{w}-x_{w}-\Delta x_{w}\right)}{\kappa_{w}}$.

$$
f\left(x_{w}+\Delta x_{w}\right)=f\left(x_{w}\right) \cdot\left[1+\frac{\frac{\Delta x_{w}}{x_{w}+\Delta x_{w}}}{\frac{\left(\kappa_{w}-x_{w}-\Delta x_{w}\right)}{\kappa_{w}}}\right]
$$

Solve the ordinary differential equation derived from (1), we find the bandwidth-type cost function:

$$
y=\frac{\Phi \cdot x_{w}}{\left(\kappa_{w}-x_{w}\right)}
$$

We can see that, the required delay parameter $\left(x_{d}\right)$ has a reversed characteristic against the required bandwidth parameter $\left(x_{w}\right)$. Therefore, we obtain the delay-type cost function:

$$
y=\frac{\Psi . \kappa_{d}}{x_{d}-\kappa_{d}}
$$

\section{Derivation of the multi-variable cost function}

We now try to derive the bandwidth-delay cost function $u\left(x_{w}, x_{d}\right)$ considering two independent QoS parameters: bandwidth $\left(x_{w}\right)$ and delay $\left(x_{d}\right)$ at the same time.

The general solution is:

$$
\frac{\left(\kappa_{w}-x_{w}\right) u}{x_{w}}=\mathcal{F}\left(\frac{\left(x_{d}-\kappa_{d}\right) u}{\kappa_{d}}\right)
$$


Equation (4) provides us a general solution comprising of a family of arbitrary functions. The specific solution is therefore:

$$
u\left(x_{w}, x_{d}\right)=\sqrt{\frac{x_{w}}{\kappa_{w}-x_{w}} \cdot \frac{\kappa_{d}}{x_{d}-\kappa_{d}}}
$$

Recursively, we can see that, the specific multi-variable cost function equals to the average multiplication of all partial cost functions:

$$
u\left(x_{1}, x_{2}, \ldots, x_{n}\right)=\sqrt[n]{\prod_{i=1}^{n} f_{i}\left(x_{i}\right)}
$$

In general, we can build a cost function for as many variables as possible given separated partial cost functions. However, while a multi-variable cost function can consider many QoS parameters at the same time, it should be noticed that the multi-variable cost function does not always give a better result than the single-variable cost function. For example, the cost function with bandwidth, delay, and packet-loss can build a better multicast tree if many peers are using wireless access network with a high packet loss rate to join the multicast tree but when most of the peers are using a wired access network with a low packet loss rate, then that three-variable cost function may build a worse multicast tree than the two-variable cost function of only bandwidth and delay. Therefore, a $\mathrm{N}$-variable cost function with $N \geq 3$ should be designed and applied with care.

\section{Video CONFERENCE SERVICE IN HETEROGENEOUS NETWORK (WIMAX+INTERNET) ENVIRONMENT}

The distributed video conferencing service can be built from an overlay network of heterogeneous networks. For example, 2 participants can participate into the video conference session from a high-speed wireless network such as WiMAX, while others participate from fixed network or from the Internet. For supporting this video conferencing service on a heterogeneous network environment, we consider the following aspects:

- Scalable video coding: Using the temporal, spatial, quality or combined scalability of this scalable video coding method[13], the video stream can adapt to the fast changing conditions and the variety of the participants' terminals in real time,

- Multi-variable cost function: The cost function used to construct the overlay network must consider both application's requirements and network conditions at the same time to provide an adaptive overlay network on heterogeneous networks,

- EvalSVC: An evaluation platform which is capable of evaluating scalable video transmission on the overlay heterogeneous environment of real WiMAX network and simulated Internet topology. The reason for proposing this evaluation platform is to show the performance of the multi-variable cost function in building the overlay network for the scalable video conferencing service in a very common case of the video conferencing service when some participants join the conference from a real WiMAX network and a large number of other participants are joining from the Internet. Using the evaluation platform, we make use of our real WiMAX networks of real WiMAX terminals, real WiMAX Base Stations (BS), real WiMAX Base Station Controllers (BSC), real WiMAX Operations and Maintenance Center (OMC) and real WiMAX core network to validate the performance cost function.

The architecture of the evaluation platform is demonstrated in Fig.2. In this architecture, each video conference participant is equipped with:

- Raw Video In/Out: The video stream from the camera in raw format before encoding and the raw video obtained from the network after decoding will go through this block,

- Video Encoder/Decoder: The raw video will be encoded/decoded in SVC format in this block,

- Video Hinter/Rebuilder: The Video Hinter will packetize SVC encoded units into RTP packets and add a hint track to the SVC bit-stream. We can consider the hint track as an in-band signaling for the SVC bit-stream. The Video Rebuilber will collect all data from sender's, receiver's dumpings and video trace files, take both the SVC encoded bit-stream and the hinted file at the sender into account and reconstruct a possibly-corrupted output SVC bit-stream at the receiver. The SVC re-builder must understand SVC NALU headers in order to properly rebuild the corrupted SVC bit-stream,

- Video Sender/Receiver: The departure and destination of the video transmission.

Two participants participate into the overlay network using a real WiMAX underlay network. N (up to 1024) other participants are participating into the same overlay network by using an underlay network of simulated Internet topology. The overlay network is constructed from the multi-variable cost function introduced in section II. The multicast measurement block calculates the common overlay metrics obtained from the evaluation platform. They are: average link stress and average end-to-end delay.

- Average link stress: defined in terms of the mean value of identical packets due to overlay forwarding, carried over a physical access link. This metric is equal to 1 for IP multicast. The lower the average link stress, the better the performance,

- Average end-to-end delay: Defines the average value of end-to-end delay on the entire overlay network.

\section{Evaluation Platform AND MEASUREMENT RESULTS}

\section{A. Settings of the evaluation platform}

To see the adaptation of the newly proposed cost function in the real network conditions, we implement a testbed based on both the Oversim-based simulation platform and two real WiMAX terminals connecting to the simulated platform by using a real WiMAX access and core network. 
TABLE I

Simulation Parameters of the SVC VIDEO CONFERENCE SERVICE ON OVERLAY NETWORK BASED ON HETEROGENEOUS NETWORK OF SIMULATED INTERNET TOPOLOGY AND REAL WiMAX NETWORK.

\begin{tabular}{|c|c|}
\hline Parameters & Values \\
\hline Purpose & $\begin{array}{l}\text { Evaluation of the newly pro- } \\
\text { posed multi-variable cost func- } \\
\text { tion for Scalable Video Confer- } \\
\text { encing service on heterogeneous } \\
\text { network (simulated Internet }+ \\
\text { real WiMAX). }\end{array}$ \\
\hline Video encoding & SNR SVC \\
\hline Video size & CIF \\
\hline Multicast & Overlay network \\
\hline $\begin{array}{l}\text { Transmission net- } \\
\text { work }\end{array}$ & $\begin{array}{l}\text { Simulated Internet topology and } \\
\text { WiMAX network }\end{array}$ \\
\hline $\begin{array}{l}\text { Number of WiMAX } \\
\text { terminals }\end{array}$ & 2 \\
\hline Service & $\begin{array}{l}\text { Application Layer Multicast for } \\
\text { scalable video conference }\end{array}$ \\
\hline $\begin{array}{l}\text { Network simulation } \\
\text { tool }\end{array}$ & Oversim \\
\hline Number of peer & $1-1024$ peers \\
\hline Underlay network & $\begin{array}{l}\text { Internet topology generated by } \\
\text { GT-ITM }\end{array}$ \\
\hline Cost functions & $\begin{array}{l}\text { - New multi-variable cost } \\
\text { function, } \\
\text { - NICE's popular cost func- } \\
\text { tion }\end{array}$ \\
\hline $\begin{array}{l}\text { Overlay measure- } \\
\text { ments }\end{array}$ & $\begin{array}{l}\text { - Average link stress, } \\
\text { - Average end-to-end delay }\end{array}$ \\
\hline
\end{tabular}

The Oversim-based simulation platform is reused from the previous simulation scenarios. The WiMAX access network comprises of an Acatel-Lucent base station (9710 CWBS). The first WiMAX terminal is an Alcatel-Lucent 9799 PCMCIA card. The second WiMAX terminal is a Sequans USB card. IEEE 802.16e-2005 state of the art Scalable OFDMA based Technology is applied. The heterogeneous network is setup as illustrated in Fig.4. Fig.3 illustrates the integration between the Oversim-based simulation platform and the WiMAX access network. This simulation scenario emulates a video conferencing service built on top of the ALM network. The participants can be divided into two groups. The first group comprises of simulated peers participating to the ALM group from the INET[14] underlay network. We use 1 or 2 peer(s) participating into the ALM group from the WiMAX network using the OMNET ++ single host underlay[15]. A tunneling interface is set up to connect between the main ALM group and the external WiMAX peer(s). Fig.2 demonstrates EvalSVC and the performance evaluation of SVC transmission on overlay network based on heterogeneous

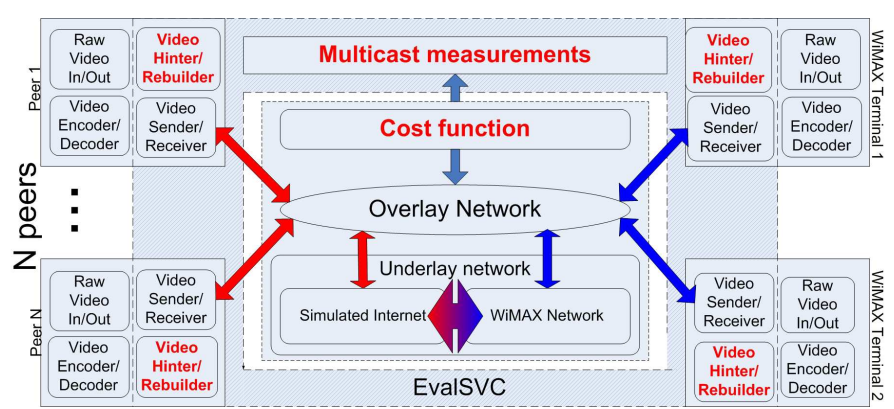

Fig. 2. EvalSVC and the performance evaluation of SVC transmission on overlay network based on heterogeneous of simulated Internet topology and real WiMAX network. The Application Layer Multicast tree is constructed using the newly proposed multi-variable cost function.

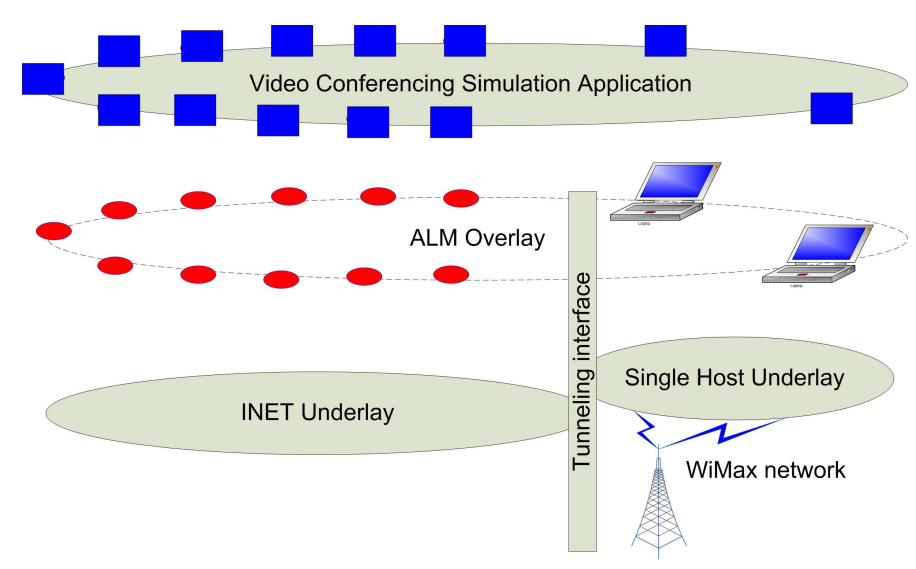

Fig. 3. Extended simulation scenario with 2 real WiMAX terminals.

of simulated Internet topology and real WiMAX network. The Application Layer Multicast tree is constructed using the newly proposed multi-variable cost function.

\section{B. Evaluation results}

Figure 5 shows that, the link stress for the WiMAX link is lower than the average value. It is because the nodes joining the ALM group from the WiMAX network are usually placed at the lower layers of the hierarchical distribution tree, therefore, the link stress on their links are usually lower than the average level of the distributed tree. However, the advantage is that, the link stress of

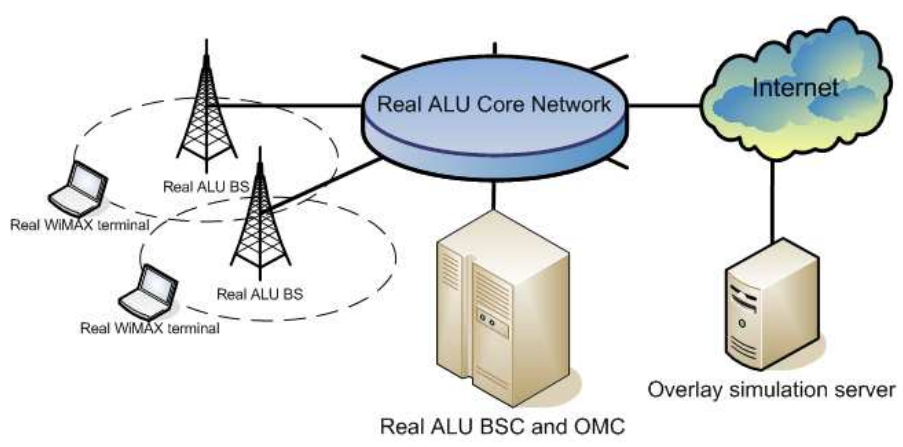

Fig. 4. Heterogenous Network: Real Alcatel-Lucent WiMAX networks. 


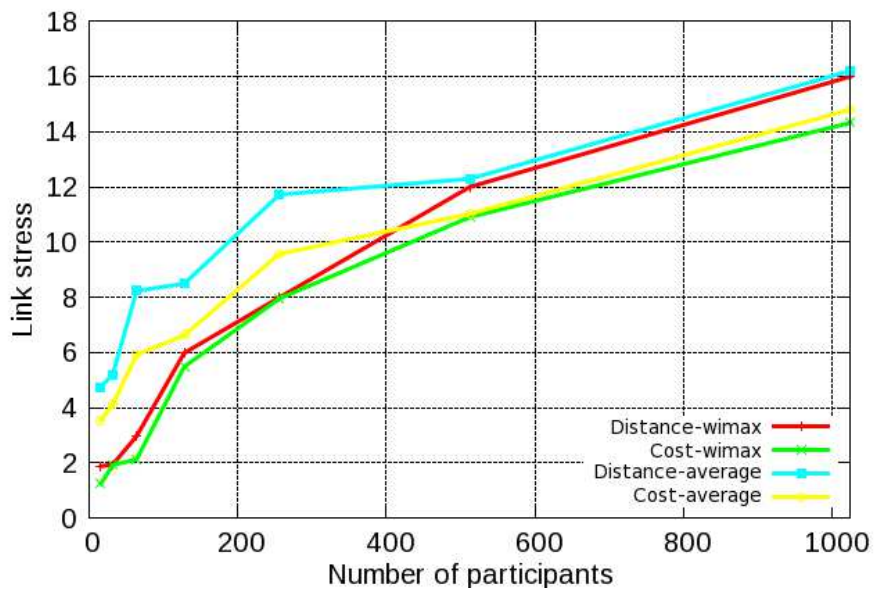

Fig. 5. Linkstress of the extended simulation scenario with 2 real WiMAX terminals.

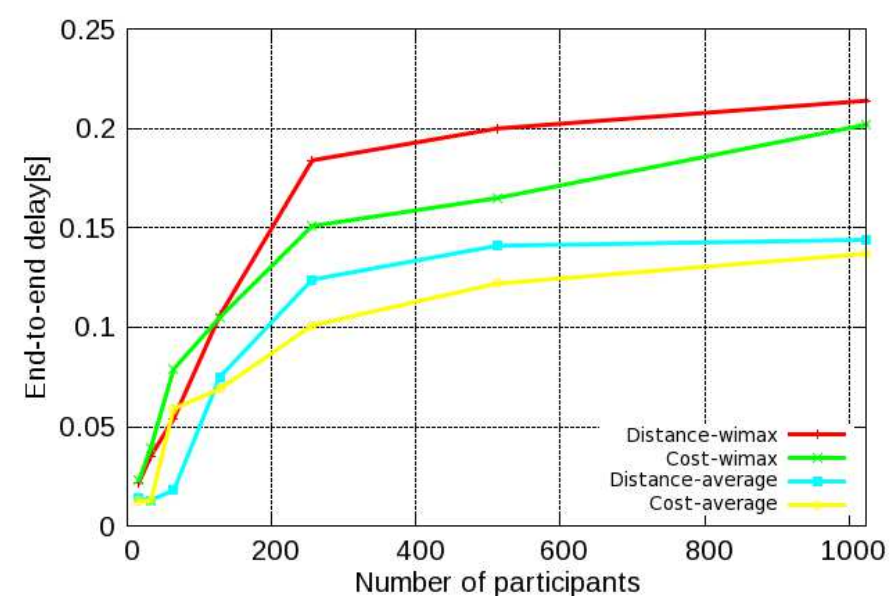

Fig. 6. End to end delay of the extended simulation scenario with 2 real WiMAX terminals.

the WiMAX link when applying the newly proposed cost function is lower than both the average level and the link stress when applying the conventional distance function. It means that, the multi-variable cost function can reduce the duplicated traffic of forwarding data on the WiMAX wireless link. This is a very important advantage of the new multi-variable cost function since the radio resource is usually limited and the lower duplicated traffic it has to transfer, the better quality it is.

Regarding the end-to-end delay performance, the result in Fig.6 shows that the WiMAX link when applying the conventional cost function has the highest end-to-end delay followed by the case when the newly proposed cost function is used.

\section{Conclusion And future works}

In this research, an evaluation platform for the scalable video conference service built from the new cost function and a heterogeneous environment of a simulated Internet topology and a real WiMAX network has been proposed. The scalable video conference service and the evaluation platform are used to evaluate the performance of the newly proposed cost function in the rapidly changing conditions of the heterogeneous network. The real measurement results collected from the real WiMAX deployment field have shown that the multi-variable cost function can adopt well to the heterogeneous network conditions and it can effectively improve the performance of the ALM-based media distributing tree. For future works, a new ALM can be designed based on the newly proposed cost function. The result can be further applied to improve the performance of any ALM algorithms who are using conventional cost functions to build their data delivery tree.

\section{Acknowledgment}

The research work is supported in part by Poseidon, a French national research project on the evaluation of multimedia services on $4 \mathrm{G}$ networks. The authors are grateful to Quang Hoang Nguyen for his contributions in building the simulation environment for EvalSVC.

\section{REFERENCES}

[1] S. E. Deering and D. R. Cheriton, "Multicast routing in datagram internetworks and extended LANs," ACM Transactions on Computer Systems (TOCS), vol. 8, no. 2, pp. 85-110, 1990.

[2] C. Diot, B. N. Levine, B. Lyles, H. Kassem, and D. Balensiefen, "Deployment issues for the IP multicast service and architecture," IEEE Network, vol. 14, no. 1, pp. 78-88, 2000.

[3] M. Hosseini, D. T. Ahmed, S. Shirmohammadi, and N. D. Georganas, "A survey of application-layer multicast protocols," IEEE Communications Surveys \& Tutorials, vol. 9, no. 3, pp. 58-74, 2007.

[4] Tien A. Le, Hang Nguyen, and Hongguang Zhang, "Multivariable cost function for Application Layer Multicast routing," in IEEE Globecom 2010 - Communications Software, Services and Multimedia Applications Symposium (GC10 - CSSMA), Miami, Florida, USA, Dec. 2010.

[5] Tien A. Le, Hang Nguyen, and Quang H. Nguyen, "Toward building an efficient Application Layer Multicast tree," in IEEE-RIVF 2010 International Conference on Computing and Telecommunication Technologies, 2010.

[6] C. Luo, W. Wang, J. Tang, J. Sun, and J. Li, "A Multiparty Videoconferencing System Over an Application-Level Multicast Protocol," IEEE Transactions on Multimedia, vol. 9, no. 8, pp. 1621-1632, 2007.

[7] Tien A. Le, Hang Nguyen, and Hongguang Zhang, "Scalable Video transmission on overlay networks," in Second International Conferences on Advances in Multimedia, Athens, Greece, June 2010, pp. 180-184.

[8] Tien A. Le, Hang Nguyen, and Hongguang Zhang, "EvalSVC An evaluation platform for scalable video coding transmission," in Consumer Electronics (ISCE), 2010 IEEE 14th International Symposium on, 2010, pp. 1-6.

[9] I. Matta and L. Guo, "On routing real-time multicast connections," in IEEE International Symposium on Computers and Communications, 1999. Proceedings, 1999, pp. 65-71.

[10] D. H. Lorenz, A. Orda, and D. Raz, "Optimal partition of QoS requirements for many-to-many connections," in IEEE INFOCOM. Citeseer, 2003, vol. 3, pp. 1670-1679.

[11] D. H. Lorenz and A. Orda, "Optimal partition of QoS requirements on unicast paths and multicast trees," IEEE/ACM Transactions on Networking (TON), vol. 10, no. 1, pp. 102-114, 2002.

[12] R. Widyono, "The design and evaluation of routing algorithms for real-time channels," International Computer Science Institute, TR-94-024, 1994.

[13] H. Schwarz, D. Marpe, and T. Wiegand, "Overview of the scalable video coding extension of the H. 264/AVC standard," IEEE Transactions on Circuits and Systems for Video Technology, vol. 17 , no. 9, pp. 1103-1120, 2007.

[14] A. Varga, "INET Framework http://inet.omnetpp.org/," 2007.

[15] A. Varga, "OMNeT++ http://www.omnetpp.org/," IEEE Network Interactive, vol. 16, no. 4, 2002. 